\title{
Drug Use before and after Initiating Treatment with Acetylcholinesterase Inhibitors
}

\author{
Anne Sverdrup Efjestad ${ }^{a} \quad$ Hege Ihle-Hansen $^{b} \quad$ Vidar Hjellvik $^{c}$ \\ Knut Engedal $^{\mathrm{b}}$ Hege Salvesen Blix ${ }^{\mathrm{c}}$ \\ ${ }^{a}$ Lørenskog Hospital Pharmacy, Lørenskog, Norway; b Ullevaal Hospital, Oslo, Norway; \\ ${ }^{c}$ Norwegian Institute of Public Health, Oslo, Norway
}

\author{
Keywords \\ Pharmacoepidemiology of dementia - Behavioral and psychological symptoms of dementia. \\ Pharmacotherapy
}

\section{Abstract}

Background/Aims: The aim was to study the prevalence of use of different drugs prescribed for behavioral and psychological symptoms of dementia in persistent users of acetylcholinesterase inhibitors (AChEls) before and after AChEl initiation, and to compare with the use in the general population. Methods: Use of antidepressants, antipsychotics, and analgesics in the 4 years before and 2 years after AChEl initiation was studied based on data from the Norwegian Prescription Database 2004-2016. Results: The prevalence of use of antidepressants and antipsychotics the year before AChEl initiation was twice the prevalence in the age-adjusted general population and continued to rise in the first 2 years after initiation of AChEls. The prevalence of weak analgesics and antipsychotics increased strongly in the last year before AChEl initiation. The increase in the use of antidepressants started at least 4 years before initiation of AChEls. Opioid use was generally lower than in the general population and was not influenced by AChEl initiation. Conclusion: Increased use of antidepressants and antipsychotics was observed both before and after initiation of AChEls and may indicate that behavioral symptoms occur in a preclinical or early phase of Alzheimer's disease. The prescription pattern of analgesics with a low use of opioids may indicate an undertreatment of pain in people with dementia. 
Efjestad et al.: Drug Use before and after Treatment with Acetylcholinesterase Inhibitors

\section{Introduction}

Morbidity and mortality due to chronic diseases like neurological and mental disorders increase in older people. More than half of all dementia cases are generally assumed to be caused by Alzheimer's disease (AD), and an increased reporting of cases is seen due to the increased number of older people $[1,2]$. Almost every fifth person will develop dementia during their lifetime [3], and the number is suggested to double in 30 years. Dementia is strongly related to age, with a prevalence in western Europe varying from about 1-2\% in the age group 65-69 years to about $25-35 \%$ in the age group 85+ [2]. According to the national Norwegian guidelines it is recommended to start treatment with acetylcholinesterase inhibitors (AChEIs) as soon as possible when the diagnosis of dementia due to $\mathrm{AD}$ is made, and the initiation of these drugs can therefore be a surrogate marker of the time of the AD diagnosis [4].

Three AChEIs are registered in Norway for symptomatic treatment of AD of mild to moderate degree; donepezil, rivastigmine, and galantamine. All three cause a general increase of acetylcholine (ACh) in all cells in the body using ACh as a transmitter substance. This gives rise to cholinergic side effects, which is of special importance in the presence of drug interactions [5], and therefore general caution must be taken to reduce the risk of adverse effects. Knowledge of comedication to AChEI treatment in people with AD may therefore be of importance.

Almost $90 \%$ of patients with AD will experience behavioral and psychological symptoms of dementia (BPSD) like depression, agitation, psychosis, apathy, or irritability during the course of dementia, and the extent increases with the severity of the dementia, especially agitation and apathy $[6,7]$. Moreover, it is possible that symptoms of apathy, depression, anxiety, and irritability can be early signs of cognitive impairment and dementia. Prescription of psychotropic drugs to these patients with $\mathrm{AD}$ in a preclinical phase could therefore be based on a wrong diagnosis and indication. In more severe stages of dementia, psychotropic drugs are often initiated to treat behavioral symptoms. However, these drugs are not particularly effective and may result in considerable side effects, especially in older people more susceptible for adverse effects of drugs $[8,9]$. Due to multimorbidity and polypharmacy, the treatment of BPSD is difficult and needs continuous clinical observation of the patients. Focused intervention to promote social engagement is an important component to enable effective antipsychotic discontinuation $[10,11]$. The modest effect of psychotropic drugs for BPSD could be due to the fact that these symptoms may have causes not related to depression, anxiety, or psychosis. Pain and discomfort may also trigger change of behavior. Psychotropic drugs are commonly involved in the treatment of BPSD in nursing homes in Norway [7, 12]. We followed patients' use of antidepressants, antipsychotics, and analgesics, which are frequently used among patients with dementia but have anticholinergic activity or other known adverse effects, interactions, and precautions [9].

AChEIs are usually initiated at the time of a diagnosis of AD, but we do not know whether this may change the prescription of psychotropic drugs. Since the first subtle signs of AD may be behavioral symptoms, psychotropic drugs would probably be prescribed. Thus, the aim was to study the pattern of drug use before and after initiation of treatment with AChEIs and compare with drug use in the general population.

\section{Materials and Methods}

Data

Drug use data were collected from the Norwegian Prescription Database (NorPD). This database covers the total population of Norway and, since 2004, information from all prescriptions dispensed at Norwegian pharmacies to individual patients are included in the 
Efjestad et al.: Drug Use before and after Treatment with Acetylcholinesterase Inhibitors

database, as well as prescriptions following treatment in inpatient clinics and discharge from hospital. It is possible to follow all individuals who purchased drugs through their national personal unique identifying number [13]. However, drugs dispensed to patients in institutions are not included in the NorPD, making us unable to follow the patients' drug use in nursing homes.

Data registered in the NorPD included sex, age, year of death (when relevant), and information on prescribed drugs dispensed. In this study, dispensed drugs represented consumed drugs. The drugs were classified according to the Anatomical Therapeutic Chemical (ATC) classification system version 2018 [14]. For each dispensed prescription we obtained the date of dispensing, medicinal product name and formulation, ATC code, number of defined daily doses, and the number of tablets/capsules/plasters (allowing us to calculate treatment periods).

We had access to individual data from the NorPD for all persons being dispensed at least one prescription of AChEIs (ATC code N06DA) in the period January 1, 2004, to December 31, 2016. In addition, for every ATC code, we had access to the yearly total number of users in the population by gender and 1-year age groups (up to and including 89 years).

\section{Study Population}

The study population consisted of all persistent (see below) AChEI users who initiated AChEI treatment between January 1, 2008, and December 31, 2013, at an age of 88 years or younger, who were alive 2 years after the year of AChEI treatment initiation, and who were registered in the NorPD the second year after AChEI initiation (not yet in a nursing home). The age limit of 88 years was set to make a comparison with the general population possible (number of users per 1-year age group in the general population was only available up to and including 89 years). The study population was stratified into four subgroups according to age at initiation. The first group, aged 37-64 years, was selected to study the presenile group (defined as onset of dementia before the age of 65). The last three groups were selected to get equal age spans (65-72, 73-80, and 81-88 years of age).

\section{Use of Drugs before and after Initiation of AChEIs}

We studied the prescriptions for the last 4 years (365-day periods) before and the first 2 years after AChEI initiation for the following drug groups: antidepressants (N06A excluding amitriptyline N06AA09, commonly prescribed for neuropathic pain), antipsychotics (N05A excluding prochlorperazine N05AB04, commonly prescribed for vertigo), and weak analgesics and opioids (ATC group N02). The day of AChEI initiation was counted as the first day of the first year (365-day period) after initiation.

\section{Incident and Persistent Use of AChEIs}

Incident use was defined as being prescribed an AChEI drug after 365 successive days with no AChEI prescriptions. According to Norwegian guidelines for dementia treatment, the treatment should be evaluated 3-6 months after initiation, and we considered users who continued treatment 8 months after initiation as persistent. Thus, an incident user was defined as persistent if any of the following was true: (i) a new prescription was given between day 210 and day 240 after initiation, (ii) drugs for at least 210 days' consumption were prescribed during the first 210 days from initiation, or (iii) the last prescription before day 210 lasted to day 210. We assumed a consumption of one tablet per day for donepezil and galantamine and two tablets per day for rivastigmine. An illustration of incident and persistent use is given in Figure 1. 
Efjestad et al.: Drug Use before and after Treatment with Acetylcholinesterase Inhibitors

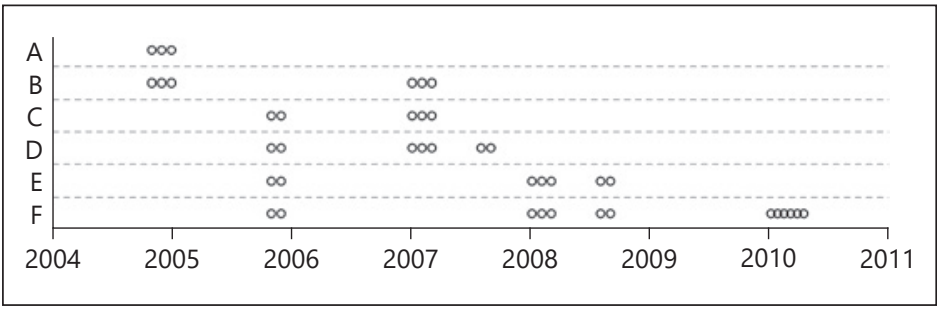

Fig. 1. Illustration of incident and persistent use. Circles indicate prescriptions. Subject $A$ is not an incident user since the first prescription came less than 365 days after January 1, 2004. B is an incident user with AChEI initiation on January 1, 2007. C and D have two new-user periods, where the last one has more prescriptions, and AChEI initiation was on January 1, 2007. D fulfills the criterion for persistent use but is not included in the study population since AChEI initiation was before January 1, 2008. E is a persistent user with AChEI initiation on January 1, 2008. F has three new-user periods, where the last one has the most prescriptions but does not fulfill the criterion for persistent use. Hence $\mathrm{F}$ is not a persistent user. Thus, all except $\mathrm{A}$ are incident users, but only $\mathrm{E}$ is a persistent user who fulfills the criteria for inclusion in the study population.

\section{Comparison with Drug Use in the General Population}

The use of, for example, antidepressants in the study population in the first year after initiation of AChEI was compared to the use in the general population (including the study population) as follows: for each of the years 2008-2013, the age- and gender-adjusted prevalence of antidepressant use in the general population was computed, using the age and gender distribution of those initiating AChEI in the actual year as reference. The overall ageand gender-adjusted prevalence in the general population was then estimated as the average of the six 1-year prevalences. When comparing antidepressant use in AChEI initiators $\mathrm{X}$ years before/after initiation with the general population, the dispensing years and the age in the general population was shifted $X$ years. For example, antidepressant use in the age group 81-88 years 4 years before initiation was compared to use in the age group 77-84 years in 2004-2009 in the general population, and antidepressant use in the same age group the second year after initiation was compared to use in the age group 82-89 years in 2009-2014 in the general population. Age-adjusted prevalence ratios (PRs) were calculated by dividing the prevalence in the AChEI users by the age- and gender-adjusted prevalence in the general population.

\section{Statistical Analysis}

$\mathrm{R}$ version 3.4.3 [15] was applied for descriptive statistics; proportions with 95\% confidence intervals (CIs) were computed for the study population. The 95\% CIs were computed using the "binom.confint" function in R with the "wilson" method. Age-adjusted prevalence was computed for the general population using the "ageadjust.direct" function in R. The 95\% CIs for the general population were very narrow and hence not shown.

\section{Results}

The study population consisted of 11,764 persistent AChEI users aged 37-88 years who initiated treatment between January 1, 2008, and December 31, 2013. The percentage of women in the four age groups was $56,56,61$, and $68 \%$, respectively, and $63 \%$ in the full study population. They were followed from 4 years (1,460 days) before initiation to 2 years $(729$ days) after initiation. 
Efjestad et al.: Drug Use before and after Treatment with Acetylcholinesterase Inhibitors

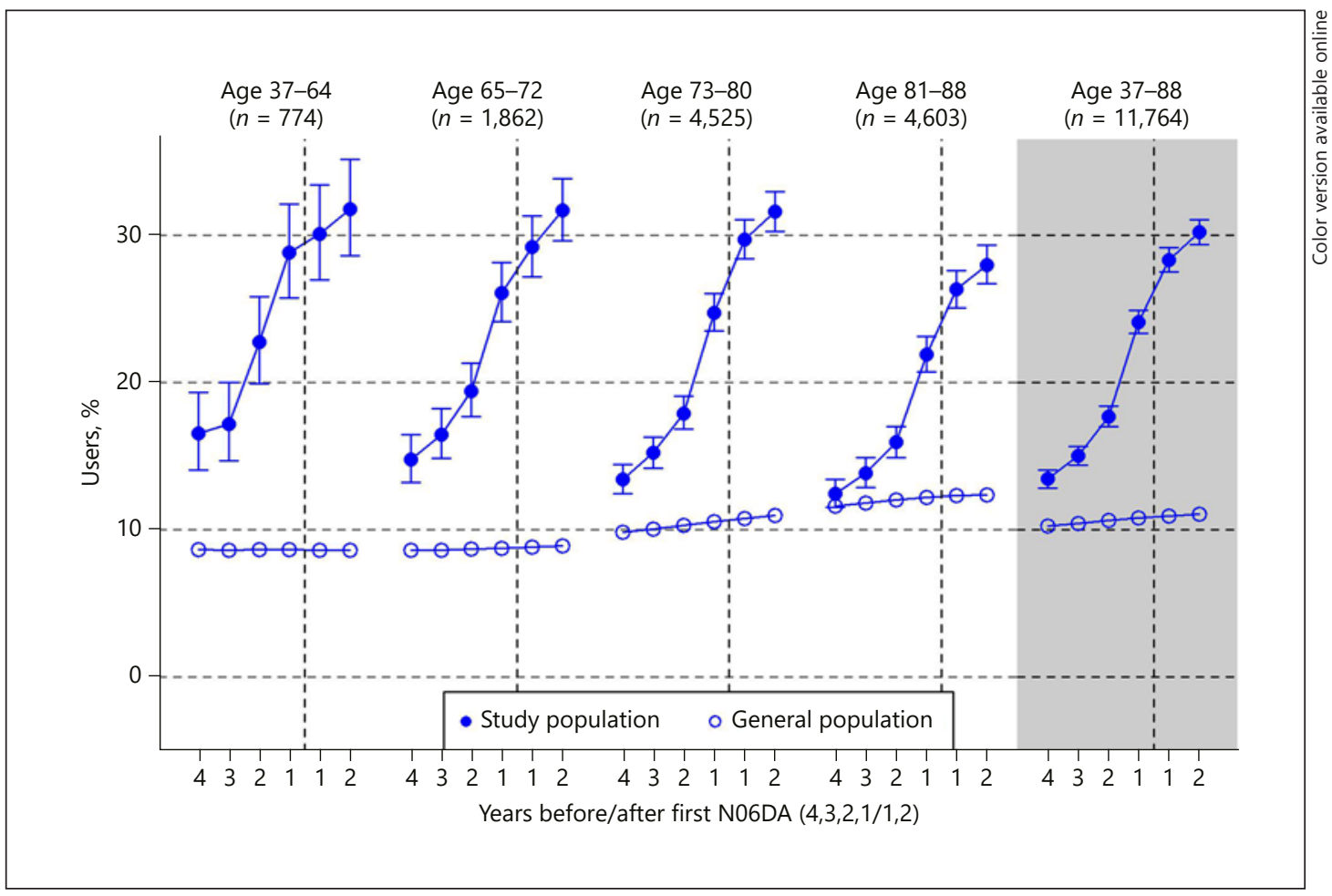

Fig. 2. Closed circles: proportion of the study population who filled at least one prescription of antidepressants in the 4 years before AChEI initiation and the 2 years after initiation, with $95 \%$ confidence intervals. Open circles: the corresponding age- and gender-adjusted proportion in the general population. Dashed vertical lines indicate AChEI initiation. The size of each age group in the study population is given on top.

The proportions of the study population and the general population receiving at least one prescription of antidepressants, antipsychotics, weak analgesics, and opioids in the 4 years before initiation of AChEI and the 2 years after initiation are shown in Figures 2-5, respectively.

The prevalence of the use of antidepressants (ATC group N06A) strongly increased in the study population over the 6-year interval from 13.5\% (95\% CI: $12.9-14.1 \%$ ) in the fourth year before initiation to $30.2 \%$ (29.4-31.1\%) in the second year after initiation. In the general population, the prevalence was quite unchanged in all age groups and increased from $10.3 \%$ to $11.1 \%$ in the age group 37-88 years (PR increased from 1.3 to 2.7) (Fig. 2). The highest prevalence was observed for AChEI users in the lower age groups. The prevalence still increased in the 2 years following AChEI introduction. In addition, as many as 87 and $82 \%$ of those who were prescribed antidepressants the year before initiation of AChEI therapy were prescribed antidepressants the first and second year after, respectively.

In the total study population, the prevalence of the use of antipsychotics (ATC group N05A) 2 years before AChEI initiation was similar to that in the general population; $3.2 \%$ (2.9-3.5) (Fig. 3), but it increased strongly in the year prior to the prescription of AChEIs to $6.0 \%$, and continued to increase in the 2 years after initiation to $9.3 \%(8.8-9.8)$ in the second year. The highest prevalence was observed in the younger age groups. The prevalence in the study population was still increasing 2 years following the prescription of AChEIs. As many as 67 and $58 \%$ of those who were prescribed antipsychotics the year before initiation of AChEI therapy were prescribed antipsychotics the first and second year after, respectively. 
Efjestad et al.: Drug Use before and after Treatment with Acetylcholinesterase Inhibitors

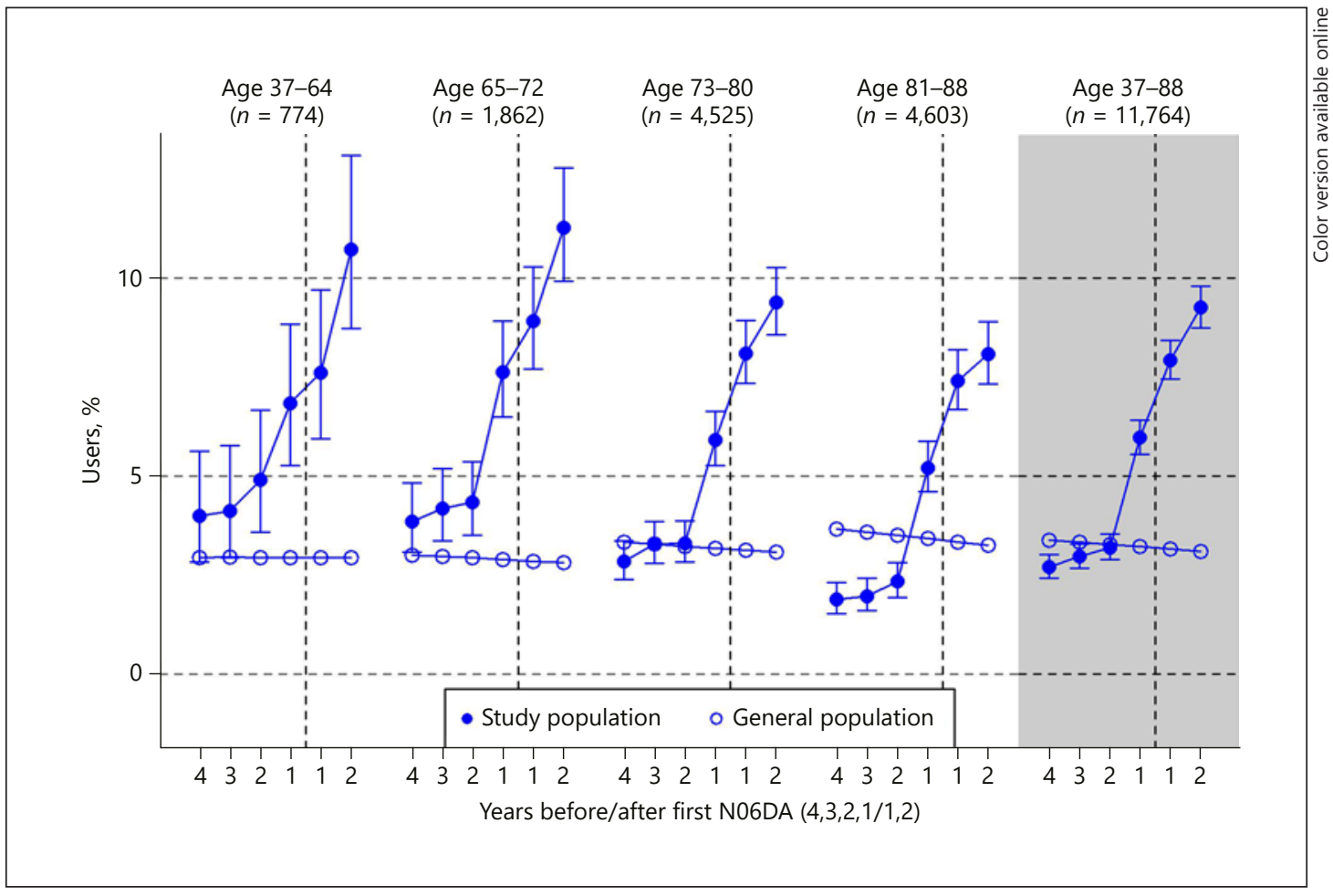

Fig. 3. Closed circles: proportion of the study population who filled at least one prescription of antipsychotics in the 4 years before AChEI initiation and the 2 years after initiation, with $95 \%$ confidence intervals. Open circles: the corresponding age- and gender-adjusted proportion in the general population. Dashed vertical lines indicate AChEI initiation. The size of each age group in the study population is given on top.

The prevalence of the use of opioids (ATC group N02A) increased slightly with age, but was lower in the study population than in the general population, mostly so in the oldest age group (Fig. 4). In the total study population, the prevalence was quite unchanged during the study period; $17.1 \%$ four years prior to AChEIs prescription and $17.2 \%$ two years after $(95 \%$ CI: 16.5-17.9). In the general population, the corresponding percentages were $19.0 \%$, increasing to $20.4 \%$ (PR decreasing from 0.90 to 0.84 ). Of those who were prescribed opioids the year before initiation of AChEI therapy, 42 and $43 \%$ were prescribed opioids the first and second year after, respectively.

In the study population, the prevalence of the use of weaker analgesics (ATC group N02B; other analgesics and antipyretics), mainly consisting of paracetamol, was similar to that in the general population (Fig. 5) until 1 year before AChEI initiation, where the prevalence in the study population increased more rapidly. In the general population, the ageadjusted prevalence increased from 11.7 to $20.0 \%$ during the study period. Corresponding percentages in the study population were $11.5 \%$ (95\% CI: $10.9-12.1)$ and $26.0 \%$, and the PR increased from 0.98 to 1.30 . The pattern was similar in the different age groups, with the lowest prevalence in the age group 37-64 years. As many as 63 and 59\% of those who were prescribed drugs in ATC group N02B (other analgesics and antipyretics) the year prior to initiation of AChEI therapy were prescribed these drugs the first and second year after, respectively. 
Efjestad et al.: Drug Use before and after Treatment with Acetylcholinesterase Inhibitors

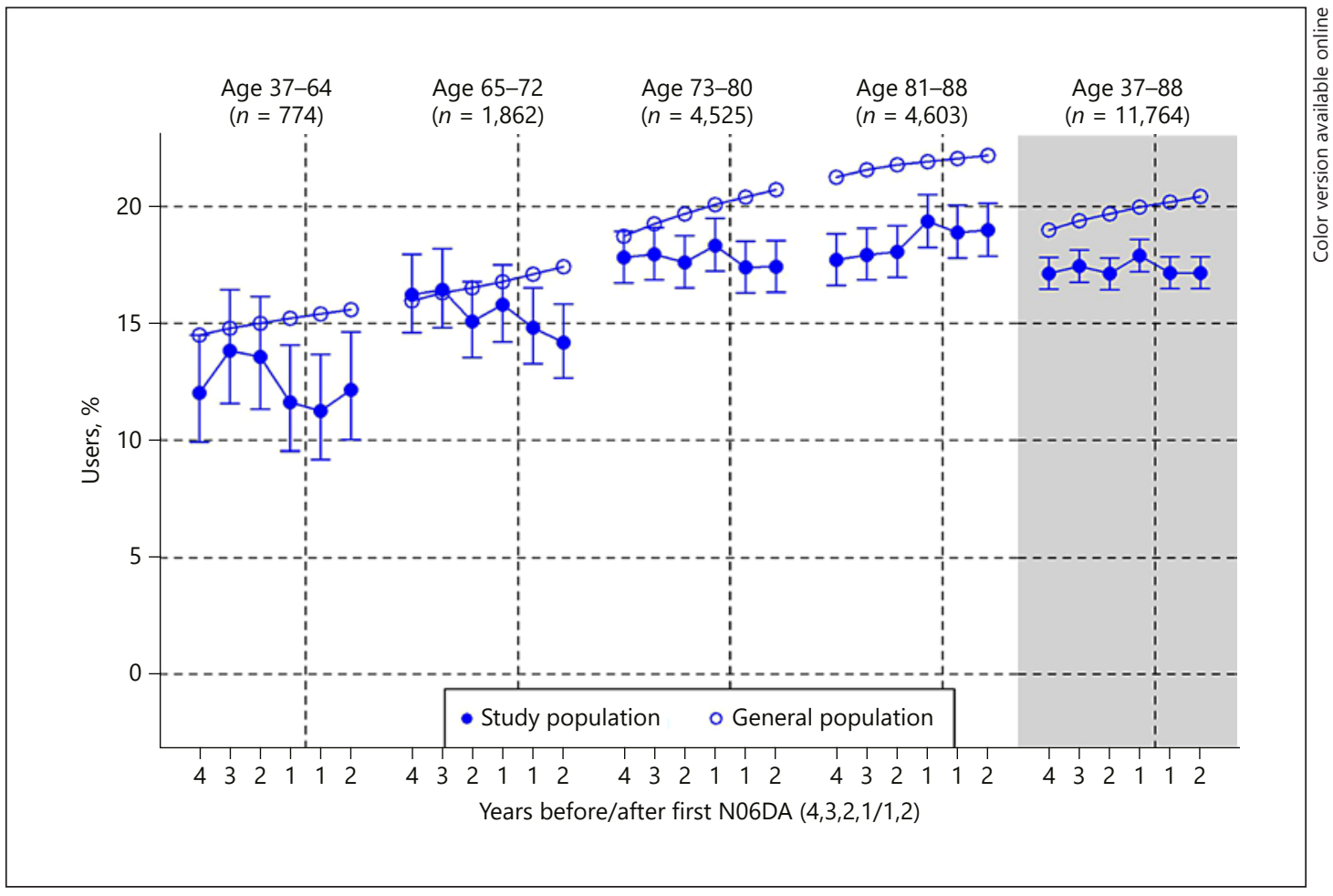

Fig. 4. Closed circles: proportion of the study population who filled at least one prescription of opioids in the 4 years before AChEI initiation and the 2 years after initiation, with $95 \%$ confidence intervals. Open circles: the corresponding age- and gender-adjusted proportion in the general population. Dashed vertical lines indicate AChEI initiation. The size of each age group in the study population is given on top.

\section{Discussion/Conclusion}

We found a marked increase in the use of antidepressants, antipsychotics, and analgesics (except for opioids) in AChEI users in the years prior to AChEI initiation, and the prevalence continued to rise in the first 2 years after initiation. The majority of those who used antidepressants the year before initiation continued after initiation. Opioid use was not influenced by AChEI initiation. Some studies have been addressing the effect of AChEIs on BPSD [6]. Our study indicates that the use of AChEIs does not reduce BPSD, as the proportion of psychotropic drug users seems to increase after initiation of treatment with AChEIs.

Antidepressants prescribed in the years before the initiation of AChEIs could indicate that depression is a preclinical or an early symptom in AD and could be a psychological response to the ongoing cognitive decline [16]. However, symptoms such as social withdrawal, problem at the workplace, apathy, reduced ability to concentrate, and forgetfulness as part of the dementia syndrome could have been misjudged as depression. Use of antidepressant medications for mood in dementia may have limited benefits but is associated with adverse outcomes [17]. However, some withdrawal studies find that some people with dementia and BPSD may benefit from antidepressants [18]. According to the Norwegian guidelines, a selective serotonin reuptake inhibitor drug should be offered as additional treatment to patients, but only when appropriate environmental psychological and/or psychotherapeutic measures have been attempted without achieving the desired effect [4]. There is insufficient evidence on the long-term safety of antidepressant use for BPSD in people with dementia [19]. Due to the limited benefits and considerable risks, antidepres- 
Efjestad et al.: Drug Use before and after Treatment with Acetylcholinesterase Inhibitors

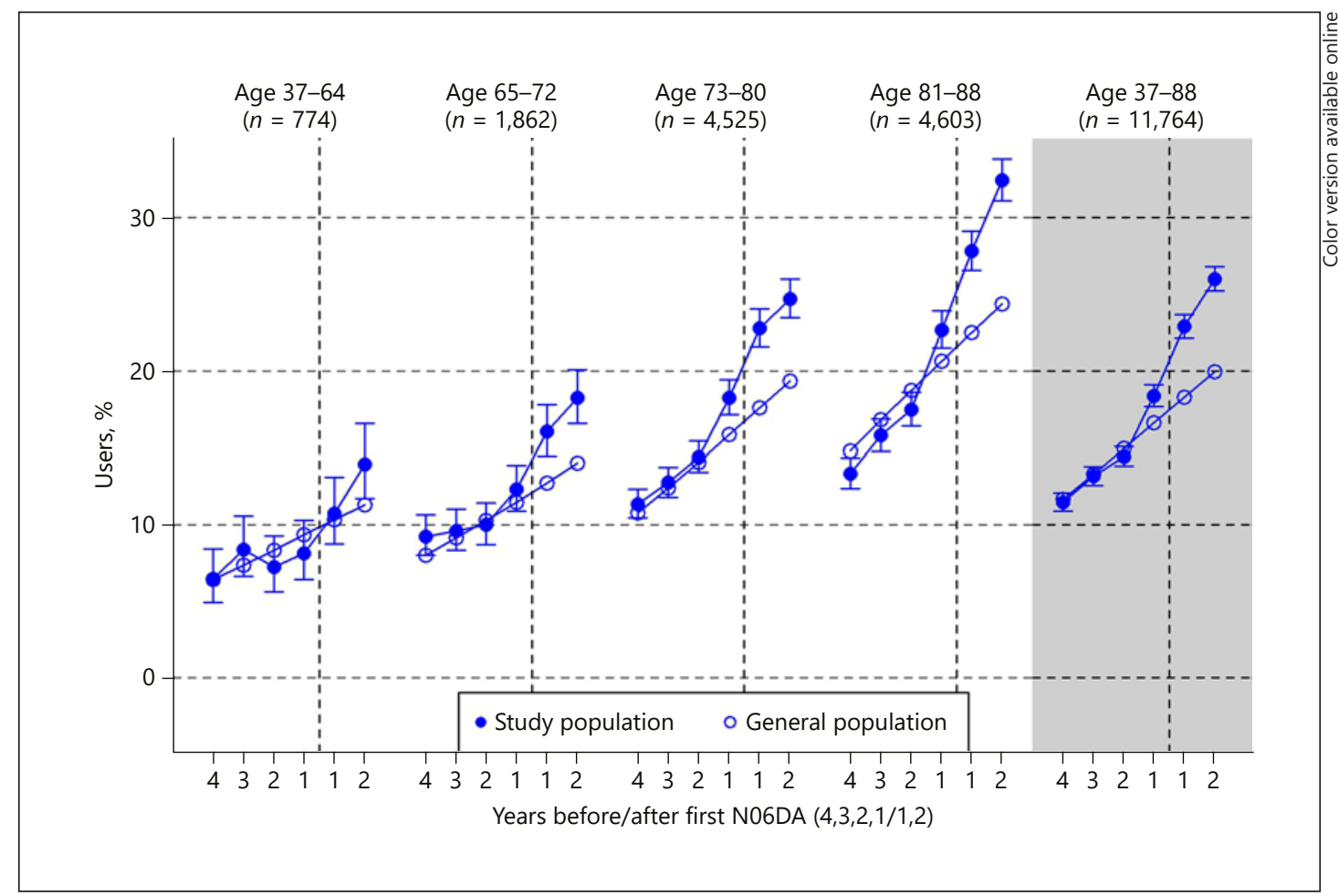

Fig. 5. Closed circles: proportion of the study population who filled at least one prescription of weaker analgesics in the 4 years before AChEI initiation and the 2 years after initiation, with $95 \%$ confidence intervals. Open circles: the corresponding age- and gender-adjusted proportion in the general population. Dashed vertical lines indicate AChEI initiation. The size of each age group in the study population is given on top.

sants should be used only if all other nonpharmacological interventions for neuropsychiatric symptoms are unsuccessful. For people living with mild to moderate dementia in combination with mild to moderate depression, psychological treatments and social activities should be preferred over antidepressants [4, 19]. The risks and benefits of different antidepressants should be carefully evaluated when these drugs are prescribed to older people $[20,21]$.

The prevalence of the use of antipsychotics increased in the study population after initiating AChEI therapy, with risks of adverse effects, interactions, and increased morbidity. The duration of antipsychotic drug treatment should be as short as possible because of the high risk of side effects, including more rapid progression of the cognitive decline [22]. Antipsychotics should therefore only be offered for people living with dementia who are either at risk of harming themselves or experiencing psychosis [4]. Apparently, psychotropic drugs are prescribed for BPSD due to lack of resources and time to implement nonpharmacological treatment approaches $[12,23]$. The highly increased fall risk in elderly individuals with AD can be the result of interaction of several factors including polypharmacy and anticholinergic side effects commonly reported with antipsychotics $[9,24]$. Patients with high anticholinergic burden more often terminate treatment with AChEIs early [25]. Of antipsychotics, the atypical substances risperidone and olanzapine currently have the best evidence for efficacy; however, the effects are modest and complicated by an increased risk of stroke [6]. In Norway, only risperidone is approved for the indication psychotic symptoms and agitation [4].

Pain is a very common manifestation in people with dementia that is often neither diagnosed nor treated. Numerous studies have shown that pain sensitivity remains largely intact 
with advanced dementia [26]. In addition, the placebo component of analgesic treatment is disrupted in AD patients [27]. Delirium, hearing and vision impairment, falls and injury, incontinence, and pain may coexist with dementia [24], and in severe dementia it may be difficult to communicate symptoms like pain. Therefore, pain and discomfort may trigger behavioral disturbances such as aggression and agitation, and also depression [28]. A clear association has been reported between pain and increased antipsychotic use, which may be related to mistreatment of pain in people with $\operatorname{AD}[28,29]$. Studies have reported frequent use of opioids in patients with dementia [30]; however, this was not the case in our study. Opioid use was not influenced by AChEI initiation. This was especially apparent in the first 2 years of the study period; the prevalence of weaker analgesics (ATC group N02B) were similar in the study group and in the general population, while a corresponding lower prevalence of opioids was observed. The increasing trend within each age group in the general population can be explained by an increased use with age. The increased prevalence of the use of weaker analgesics (ATC group N02B) observed from 2 years before the introduction of AChEIs will probably not compensate for the reduced use of opioids. The total pattern of analgesic prescription among the AChEI users may indicate an undertreatment of pain in people with dementia. The reason for the possible undertreatment may be that people with dementia underreport pain as a symptom, in addition to the prescribers' fear of adverse effects of opioids [26]. Paracetamol should always be considered the first-line therapy for pain. In case of moderate to severe pain, low and titrated dosages of an opioid are recommended, and transdermal buprenorphine may be an option [28]. Good evidence indicates that undertreatment of pain is a greater risk factor for the development of delirium than the use of opioids [31]. When prescribing opioids for treating pain in dementia, great consideration should be given to drug selection and dosing frequency [26]. Particularly in older people with dementia, pharmacological treatment should be initiated with great caution. Starting dosage(s) should be low and titrated to response [28].

Data from the NorPD give us a unique opportunity to study drug use patterns, highlighting changes over time in the selected drug groups. The large sample size is a strength in our study. However, patients in institutions are not included in NorPD and therefore not included in the present study. The NorPD does not include data on the use of over-the-counter drugs and herbal drugs. This may lead to an underestimation of weak analgesics in our study as paracetamol in small pack sizes is available as an over-the-counter drug. AChEIs are mainly prescribed for the treatment of $\mathrm{AD}$; however, we did not have information with regard to the clinical presentation of the dementia diagnoses or about the etiology or comorbidities. We did not study benzodiazepines since these drugs are, according to approved indications, mainly used for anxiety and less prescribed for BPSD in Norway. However, as the use of benzodiazepines is rather frequent in this age group, it would have been of interest to study this drug class in more detail. Purchased drugs were used as a surrogate for consumed drugs and may cause overestimation. However, medicine adherence in AChEI users is expected to be good, as caregivers are usually responsible for drug management for patients with dementia.

In conclusion, the study group receiving AChEIs differed from the general population with regard to prevalence of the use of antidepressants, antipsychotics, and analgesics. An increased use of antidepressants and antipsychotics was observed both before and after initiation of AChEIs, which may indicate that behavioral symptoms occur in a preclinical or early phase of $\mathrm{AD}$. The total pattern of analgesic prescription may indicate an undertreatment of pain in people with dementia. Evaluation of depressive and other noncognitive symptoms, as well as pain-related conditions, in people with dementia in an early phase should be prioritized to avoid the unfortunate use of psychotropic drugs and misinterpretation of early symptoms of AD. After the initiation of AChEIs, the indication for continuous use of antidepressants and antipsychotics should be considered. 
Efjestad et al.: Drug Use before and after Treatment with Acetylcholinesterase Inhibitors

\section{Acknowledgments}

We thank the Norwegian Institute of Public Health for help in obtaining data from the NorPD and the Hospital Pharmacies Enterprise, South Eastern Norway, for support.

\section{Statement of Ethics}

The NorPD generated pseudonymous files for research purposes, as regulated by Norwegian law for health registers, hence there was no demand of additional approval by the ethics committee.

\section{Disclosure Statement}

The authors have no financial or any other kind of personal conflicts in relation to this paper.

\section{Funding Sources}

The authors have not received any funding beyond the usual salaries from their institutions.

\section{References}

1 Prince MJ, Wu F, Guo Y, Gutierrez Robledo LM, O'Donnell M, Sullivan R, et al. The burden of disease in older people and implications for health policy and practice. Lancet. 2015 Feb;385(9967):549-62.

2 Hjellvik V, Engedal K, Handal M, Flaten TP, Langballe EM, Selmer R, et al. Dementia in the National Cause of Death Registry in Norway 1969-2010. Nor Epidemiol. 2012;22(2):217-24.

3 Skirbekk V, Strand BH, Tambs K. Dementia in Norway - Public Health Report 2014. Available from: https:// wwwfhino/en/online-publications/public-health-report-2014.

4 National professional guideline dementia [online]. The Norwegian Directorate of Health [accessed 2018 November 2]. Available from. www.helsedirektoratet.no17.12.2017.v.3.1.

5 Alzheimer's disease: beware of interactions with cholinesterase inhibitors. Prescrire Int. 2006 Jun;15(83): 103-6.

6 Sink KM, Holden KF, Yaffe K. Pharmacological treatment of neuropsychiatric symptoms of dementia: a review of the evidence. JAMA. 2005 Feb;293(5):596-608.

7 Selbaek G, Kirkevold $\emptyset$, Engedal K. The course of psychiatric and behavioral symptoms and the use of psychotropic medication in patients with dementia in Norwegian nursing homes-a 12-month follow-up study. Am J Geriatr Psychiatry. 2008 Jul;16(7):528-36.

8 Azermai M, Petrovic M, Elseviers MM, Bourgeois J, Van Bortel LM, Vander Stichele RH. Systematic appraisal of dementia guidelines for the management of behavioural and psychological symptoms. Ageing Res Rev. 2012 Jan;11(1):78-86.

9 Pasqualetti G, Tognini S, Calsolaro V, Polini A, Monzani F. Potential drug-drug interactions in Alzheimer patients with behavioral symptoms. Clin Interv Aging. 2015 Sep;10:1457-66.

10 Ballard C, Orrell M, YongZhong S, Moniz-Cook E, Stafford J, Whittaker R, et al.. Impact of antipsychotic review and nonpharmacological intervention on antipsychotic use, neuropsychiatric symptoms, and mortality in people with dementia living in nursing homes: a factorial cluster-randomized controlled trial by the wellbeing and health for people with dementia (WHELD) program. Am J Psychiatry. 2016 Mar;173(3):252-62.

11 Davies R. Gill Livingston: transforming dementia prevention and care. Lancet. 2017 Dec;390(10113):2619.

12 Fog AF, Kvalvaag G, Engedal K, Straand J. Drug-related problems and changes in drug utilization after medication reviews in nursing homes in Oslo, Norway. Scand J Prim Health Care. 2017 Dec;35(4):329-35.

13 FOR-2003-10-17-1246. Regulations concerning the collection and processing of health data in the prescription database [online] [accessed 2018 November 2]. Available from. www.lovdata.no. 20.10.2003. 
14 WHO Collaborating Centre for Drug Statistics Methodology. ATC Classification Index with DDDs 2018. Centre for Drug Statistics and Methodology, Oslo 2018 [accessed 2018 November 2]. Available from: https:// wwwwhoccno/atc_ddd_index/.

15 R Core Team. A language and environment for statistical computing. R Foundation for Statistical Computing, Vienna, Austria [accessed 2018 November 2]. Available from: URL http://wwwR-projectorg/.

16 Mirza SS, de Bruijn RF, Direk N, Hofman A, Koudstaal PJ, Ikram MA, et al. Depressive symptoms predict incident dementia during short- but not long-term follow-up period. Alzheimers Dement. 2014 Oct;10(5 Suppl):S323-9.

17 Mueller C, Huntley J, Stubbs B, Sommerlad A, Carvalho AF, Perera G, et al. Associations of neuropsychiatric symptoms and antidepressant prescription with survival in Alzheimer's Disease. J Am Med Dir Assoc. 2017 Dec;18(12):1076-81.

18 Bergh S, Selbaek G, Engedal K. Discontinuation of antidepressants in people with dementia and neuropsychiatric symptoms (DESEP study): double-blind, randomized, parallel group, placebo controlled trial. BMJ Open. 2012;344:e1566.

19 Bui Q. Antidepressants for agitation and psychosis in patients with dementia. Am Fam Physician. 2012 Jan; 85(1):20-2.

20 Coupland C, Dhiman P, Morriss R, Arthur A, Barton G, Hippisley-Cox J. Antidepressant use and risk of adverse outcomes in older people: population based cohort study. BMJ. 2011 Aug;343:d4551.

21 Ford AH, Almeida OP. Management of depression in patients with dementia: is pharmacological treatment justified? Drugs Aging. 2017 Feb;34(2):89-95.

22 Helvik AS, Šaltytė Benth J, Wu B, Engedal K, Selbæk G. Persistent use of psychotropic drugs in nursing home residents in Norway. BMC Geriatr. 2017 Feb;17(1):52.

23 Koponen M, Taipale H, Tanskanen A, Tolppanen AM, Tiihonen J, Ahonen R, et al. Long-term use of antipsychotics among community-dwelling persons with Alzheimer?s disease: A nationwide register-based study. Eur Neuropsychopharmacol. 2015 Oct;25(10):1706-13.

24 Heun R, Schoepf D, Potluri R, Natalwala A. Alzheimer's disease and co-morbidity: increased prevalence and possible risk factors of excess mortality in a naturalistic 7-year follow-up. Eur Psychiatry. 2013 Jan;28(1): 40-8.

25 Sverdrup Efjestad A, Ihle-Hansen H, Hjellvik V, Blix HS. Comedication and treatment length in users of acetylcholinesterase inhibitors. Dement Geriatr Cogn Disord Extra. 2017 Feb;7(1):30-40.

26 Widera E, Covinsky KE. What are appropriate palliative interventions for patients with advanced dementia? In: Goldstein NE, Morrison RS, editors. Evidence-based practice of palliative medicine. Philadelphia: Saunders; 2013.

27 McAuliffe L, Brown D, Fetherstonhaugh D. Pain and dementia: an overview of the literature. Int J Older People Nurs. 2012 Sep;7(3):219-26.

28 Flo E, Husebø B. Treating pain in dementia-are we good enough? J Pain Palliat Care Pharmacother. 2015 Sep; 29(3):303-4.

29 Rajkumar AP, Ballard C, Fossey J, Orrell M, Moniz-Cook E, Woods RT, Murray J, Whitaker R, Stafford J, Knapp M, Romeo R, Woodward-Carlton B, Khan Z, Testad I, Corbett A. Epidemiology of pain in people with dementia living in care homes: Longitudinal course, prevalence, and treatment implications. J Am Med Dir Assoc. 2017 May;18(5):453.e1-e6.

30 Jensen-Dahm C, Gasse C, Astrup A, Mortensen PB, Waldemar G. Frequent use of opioids in patients with dementia and nursing home residents: A study of the entire elderly population of Denmark. Alzheimers Dement. 2015 Jun;11(6):691-9.

31 Farris G, Mattison ML. Delirium. Hosp Med Clin. 2014;3(1):e85-92. 